\title{
Stigma and Lack of Social Support Contribute to Loss-To-Follow-Up in a Cervical Cancer Prevention Program in Western Kenya
}

\section{Carissa Novak ( $\nabla$ carissa.ashley.novak@gmail.com )}

University of North Carolina at Chapel Hill https://orcid.org/0000-0002-7446-4313

\section{Bonnie Kaiser}

University of California San Diego

\section{Lawrence Park}

Duke Global Health Institute

\section{Sandra Yvonne}

Kenya Medical Research Institute

\section{Saduma Ibrahim}

Kenya Medical Research Institute

\section{Breandan Makhulo}

Kenya Medical Research Institute

\section{Megan Huchko}

Duke Global Health Institute

\section{Research article}

Keywords: HPV, Cervical Cancer, Low-Resource, Cryotherapy, Treatment, Self-testing, Obstetrics, Gynecology, Stigma, Social support

Posted Date: May 8th, 2020

DOI: https://doi.org/10.21203/rs.3.rs-26138/v1

License: (a) (1) This work is licensed under a Creative Commons Attribution 4.0 International License. Read Full License 


\section{Abstract \\ Background}

While highly preventable, cervical cancer remains a leading contributor to the global burden of cancer among women globally, with disproportionate impacts in Sub-Saharan Africa. Human papillomavirus (HPV) testing is a cost-effective screening strategy with the potential to increase screening uptake; however, the two-visit requirement often leads to substantial loss-to-follow-up (LTFU) for treatment. Understanding modifiable factors that contribute to LTFU is essential to ensuring maximal program impact.

\section{Methods}

We carried out a mixed-methods study to understand factors related to LTFU following an HPV-based cervical cancer screening campaign in rural Western Kenya. We randomly selected participants among women testing HPV positive as part of an existing intervention study to complete quantitative surveys, seeking equal numbers of treated and LTFU women. A subset of women from both groups was selected for in-depth interviews (IDIs).

\section{Results}

Sixty-one treated and 39 LTFU women completed the quantitative survey, and 10 women from each group completed IDIs. Cost of transportation and distance to the hospital were barriers among all women, who often depended on their partners to facilitate this. Among treated women $67 \%(n=41)$ reported that their peers knew their HPV test result, compared to $38 \%(n=15)$ among LTFU women $(p=.007)$. Stigma and lack of partner and peer support emerged as strong barriers among LTFU women. Women proposed peer encouragement, including men in educational sessions, bringing facilities closer, and providing transportation as facilitators to treatment access.

\section{Conclusion}

Lack of partner and social support emerged as key barriers to treatment uptake among LTFU women. Future interventions should explore stigma reduction, male involvement, peer support, and alternative transportation options as potential facilitators to treatment seeking.

\section{Background}

Cervical cancer is a preventable disease that remains a leading form of cancer in women worldwide. Cervical cancer is the fourth most frequently diagnosed and fourth leading cause of cancer death in women globally, with an estimated 570,000 cases and 311,000 deaths in 2018.[1] Over 440,000 cases 
occur in low- and middle-income countries.[2] Sub-Saharan Africa carries a high burden of deaths, with a mortality rate of $85 \%$.[3] In Kenya alone, there are over 4,800 new cervical cancer cases and 2,000 deaths due to cervical cancer each year.[2] A lack of effective screening and treatment strategies is a leading cause of the high burden of cervical cancer deaths in low-income countries.[4]

Nearly all cases of cervical cancer are caused by human-papillomavirus (HPV), and while most HPV infections resolve naturally, persistent infection can lead to precancerous lesions that will lead to invasive cancer if undetected and treated.[5] It is essential that secondary prevention methods of screening and treatment be implemented at the population level. The World Health Organization and the Kenya Ministry of Health recommend HPV screening as a feasible and cost-effective strategy for early detection in lowresource settings.[6-8] Low-resource countries such as Kenya have had to adjust their screen and treat strategies and have moved toward HPV testing, followed by treatment with cryotherapy for HPV positive women.[5] While the screen and treat strategy is a vastly simplified screening protocol compared to that used in high-resource countries, there remain significant barriers for follow-up visits for treatment.

Despite simplified screening processes, there are still significant barriers to treatment, particularly in rural settings. Screening programs will remain ineffective at reducing the cervical cancer burden if follow-up and treatment are insufficient or inaccessible.[9] While it is generally recognized that reducing number of required visits is helpful in reducing loss to follow-up, little research has been conducted on strategies aimed at improving attendance at follow-up visits for women with positive screening results.[5] Behavioral and psychosocial factors such as stigma and social support, have been understudied as the attention has been focused primarily on logistical and access to healthcare barriers. Identifying facilitators of treatment access for HPV positive women could inform future strategies for promoting linkage to treatment.

To address this evidence gap, this study aimed to quantitatively identify factors that affect treatment access among screen-positive women who either sought treatment or were lost to follow-up (LTFU). Secondly, this study aimed to qualitatively explore the barriers and facilitators to treatment-seeking behavior among HPV positive women. Findings from this study have the potential to inform more effective screen and treat strategies implemented at the population level in low-income countries.

\section{Methods}

This mixed-methods study exploring treatment-seeking behavior among HPV-positive women was integrated into an ongoing cluster-randomized trial of implementation strategies for cervical cancer screening among women in rural western Kenya. In the parent study, women were offered screening for HPV using self-collected swabs in either community health campaigns or clinics.[10] Any woman who tested positive for HPV was referred to the County Hospital for treatment with cryotherapy, according to WHO guidelines. For the study described in this paper, we administered a treatment acquisition behavior survey among $100 \mathrm{HPV}$ positive women, followed by in-depth interviews (IDIs) with twenty women to assess barriers and facilitators to uptake of care. 


\section{Setting}

The study took place in rural villages throughout Migori County, located within Nyanza Province in Western Kenya. In the main study, twelve communities were randomized to offer screening to women aged 25-65 in either government health facilities (control) or community-health campaigns (intervention).

\section{Participants}

For this study, we defined lost to follow-up (LTFU) as women who had initially engaged in care through screening and received their HPV test result and had not yet accessed treatment 3 months after diagnosis. Women who tested positive for HPV in the main study - and who had not yet participated in other in-depth interviews - were randomly selected, stratified by treatment status with the goal of recruiting 50 who had acquired treatment and 50 who were LTFU. We then conducted in-depth interviews (IDIs) with 10 women in each group. Women were purposively selected for IDIs based on their survey responses, ensuring interviews included women with diverse experiences regarding treatment seeking.

\section{Measurements}

The survey questionnaire and IDI guide were translated from English to Dholuo, the local language. For the questionnaire, a Dholuo-speaking female research assistant obtained verbal consent from women surveyed by phone and written consent from women surveyed in person; surveys were conducted verbally with research participants. The survey questionnaire consisted of 82 questions covering demographic characteristics, alcohol and drug use, mental health, abuse history, peer network beliefs, and treatment.

A Dholuo-speaking female research assistant conducted in-person IDIs in a private space using a semistructured interview guide. Consent was reaffirmed. The guide included 15 questions with the following subsections: demographic characteristics, thoughts and experiences regarding treatment-seeking, treatment-seeking challenges, and suggestions for improving treatment uptake. Both the survey and indepth interviews were piloted among the research assistants prior to conducting with participants. The IDIs were recorded, transcribed, and then translated from Dholou to English.

\section{Data analysis}

Data from the treatment acquisition behavior survey were imported into SAS for analysis. Descriptive statistics, including frequency tables and crosstabs for categorical variables and distributions for continuous variables, were used to describe the demographic and other characteristics of the sample. Comparisons between treated and LTFU women were presented, including $p$-values for significant values or values trending towards significance. P-values were obtained using Wilcoxon test for continuous variables and Fishers Exact test for categorical variables.

Data were analyzed with Nvivo. The codebook was created using both structural codes developed using the semi-structured interview guide, and inductive codes, developed by reviewing interview transcripts until saturation was reached. The analysis process focused on developing code-by-code comparisons of 
women who were treated and women who were LTFU for treatment. Coded segments were reviewed for each theme separated by group (treated and LTFU), with data read closely to develop a thick description of the data comparing treated and LTFU groups.

\section{Ethical considerations}

The study received ethical approval from the Kenya Medical Research Institute (KEMRI) and Duke University.

\section{Results}

Description of the sample

The survey was conducted with $100 \mathrm{HPV}$ positive women, 61 treated and 39 LTFU. From this group, 10 treated women and 10 LTFU women participated in IDIs. We were unable to reach our target of 50 treated and 50 LTFU surveyed women, primarily due to the inherent challenge of contacting the LTFU population. Therefore, we continued contacting women from the treated group until we reached our target of $100 \mathrm{HPV}$ positive women. The median age of the women was 40 and 35, for treated women and LTFU women, respectively. There were no significant differences in any of the demographic characteristics based on treatment status (see Table 1). Several facilitators and barriers to treatment seeking arose from the data, including social support and peer encouragement, partner support, and stigma, among several other logistical barriers.

Table 1: Sample demographics and reproductive characteristics 


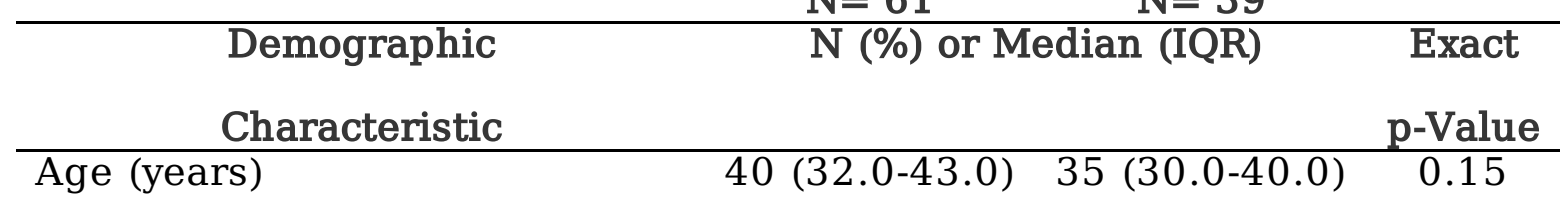

Relationship Status

$\begin{array}{lcc}\text { Married } & 37(60 \%) & 24(61 \%) \\ \text { Relationship Living Separate } & 1(2 \%) & 0(0 \%) \\ \text { Single } & 1(2 \%) & 1(3 \%) \\ \text { Widowed/Divorced } & 22(36 \%) & 14(36 \%)\end{array}$

Education Level

0.68

Primary

$45(74 \%) \quad 34(87 \%)$

Secondary

$14(23 \%) \quad 4(10 \%)$

Tertiary/College

$2(3 \%)$

$0(0 \%)$

0.38

Other

$0(0 \%)$

$1(3 \%)$

Occupation Earning Income

Yes

$25(41 \%)$

$14(36 \%)$

No

$36(59 \%)$

$25(64 \%)$

Occupation Type ( $\mathrm{N}=39)$

Professional/Managerial

$2(8 \%)$

$0(0 \%)$

0.18

Agriculture and Fishing

$5(20 \%)$

$3(22 \%)$

Manual

$4(16 \%)$

$0(0 \%)$

Sales and Services

Other

$13(52 \%)$

$9(64 \%)$

$1(4 \%)$

$2(14 \%)$

Primary Income Earner ( $\mathrm{N}=39)$

Yes

$14(56 \%)$

0.68

No

$11(44 \%)$

$11(79 \%)$

$3(21 \%)$

Religion

Christian

$61(100 \%)$

$39(100 \%)$

0.20

Has Children

Yes

$58(95 \%)$

$36(92 \%)$ 
Number of Children

*IQR - Interquartile range

*LTFU - Lost to follow-up

\section{Logistical barriers}

Cost of transportation and distance to the health facility were the most commonly reported barriers among all women; however, these did not differ significantly or meaningfully between treated and LTFU women. Additionally, while cost of transportation and distance to the health facility were both most frequently mentioned in the IDIs, cost of transportation was the predominant barrier selected in the survey. In the survey, women who sought treatment were asked what ways (if at all) treatment made them feel nervous or uncomfortable. Of the 69 women who sought treatment, $49 \%(n=30)$ reported there were none, and $27 \%(n=17)$ reported they were worried about the cost of transportation to the health facility. Among LTFU women, cost was the primary reason for delaying in seeking treatment, with $62 \%(n=24)$ of women reporting this barrier. Approximately $5 \%$ of women $(n=2)$ cited travel distance and $5 \%(n=2)$ cited not knowing the treatment place as being barriers. The remaining $28 \%(n=11)$ cited other reasons such as logistical barriers, doctor and nurse strike, mechanical problems at treatment facility, fear of partner learning their HPV status, and losing the phone number needed to schedule the treatment appointment (Table 2). The IDIs also identified the biggest challenges faced by all women as the cost of transportation and distance to the treatment facility. Treated and LTFU women agreed the trip to Migori Hospital was long and costly.

Table 2: Logistical and other barriers to treatment 


\section{Treated $(\mathrm{N}=61)$}

In which ways did treatment make you feel nervous or uncomfortable

None

Worried about cost of transportation

Thought it would be painful

I was not sure it would work

Embarrassed of having a pelvic exam

Fear people would learn I am HPV positive

Other (fear b/c of how people were talking about treatment)

The trip from home to treatment facility was long

It was difficult to get money for trip to treatment facility

\section{LTFU $(\mathrm{N}=39)$}

Main reason for delaying seeking treatment

Cost

Other

Travel Distance

Not knowing treatment place

\section{Social support and peer encouragement}

Treated women were more likely than LTFU women to share their HPV test result with their peers $(67 \%$ vs. $38 \%, p=0.007)$ and their family $(61 \%$ vs. $38 \%, p=0.04)$. They also reported greater comfort levels discussing HPV results compared to LTFU women $(p=0.01)$ and were more likely than LTFU women to know whether their peers tested ( $82 \%$ vs. $69 \%, p=0.17)$ and what their HPV status was $(90 \%$ vs. $67 \%$, $\mathrm{p}=0.027)($ Table 3$)$.

In the IDIs, nearly all treated women reported they knew friends or family who were diagnosed with HPV, and all those women also reported that those friends or family members sought treatment and 
shared their experience with them. In contrast, very few LTFU women knew friends or family who were diagnosed with HPV, and therefore knew few people who had sought treatment. In both groups, the person they knew who had experienced screening and/or treatment was likely to be a close friend or a cowife in a polygamous household; other relatives were rarely mentioned.

Treated women spoke about their friends' and families' experiences in seeking and experiencing treatment in detail: they described why they decided to seek treatment, the method of transportation used, and almost all said they had a painless and simple procedure, and that they had manageable levels of discharge post-treatment. While LTFU women reported that people they knew were satisfied with treatment, they were more vague in their responses, with many responses centered around getting treatment "to improve their health."

Treated women cited peer encouragement as a key motivator for seeking treatment and said that women coordinated to go to treatment together and talked openly about taking control of their health and stopping the disease before it becomes deadly. A treated woman said, "I used to share a lot with a friend who encouraged me that we should use that opportunity productively. So we made a decision to participate and get treated." Another treated woman said, "We normally story [talk] in a group, and during that period we encourage one another to seek treatment, not to be a victim of risks associated with HPV." There were several LTFU women who had been invited by other women to join them for treatment but did not have money for transport: "Yes, we did encourage ourselves and made a promise that if possible we will go for treatment as a group. But we allowed those of us who were ready to go. That is, if you have [money for] transport ready." Overall, treated and LTFU women described markedly different experiences regarding peer encouragement.

During the IDIs, treated women more commonly said they have a trusted friend or family member that they can lean on for support. Social support manifest in multiple ways; women referred to having someone to talk to about their health, as well as someone to help with household chores and duties while away for treatment, and even had someone to attend treatment with. There were very few LTFU women who cited having someone to lean on for support, or that they had a trusted friend or family member that could help in the coordination of attending treatment. A LTFU woman referred to feeling alone in this endeavor and suggested that her socioeconomic status may have played a role, saying, "Yes, I have 
relatives and friends who are well off, but the haves never mixed well with the have not. They make promises which they never fulfill, so there is no need, we have to survive on our own." Nearly all treated women expressed their interest in sharing their own experience in a group setting to encourage other women to go for treatment, and nearly all LTFU women said they were interested in hearing from those who had been through the treatment process.

Table 3: Peer network beliefs and behaviors among women who sought treatment and LTFU women

\section{Peer Network Beliefs}

Does partner know HPV result

Yes

No

Peers know your HPV result

Family knows your HPV result

Do you feel comfortable talking about result with close friends/family

Yes comfortable with everyone

Comfortable with some people

Not comfortable with anyone

Did any peers get tested for HPV

Yes

No

Do not know

Knew peers' HPV status
Treated LTFU Exact

$\mathrm{N}=61 \quad \mathrm{~N}=39 \quad \mathrm{p}-$

Value

0.22

$\begin{array}{cc}39 & 20(51 \%) \\ (64 \%) & 19(49 \%) \\ 22 & \end{array}$

(36\%) $15(38 \%) \quad 0.007$

$41 \quad 15(38 \%) \quad 0.0404$

(67\%)

37

(61\%)

(33.3\%)

13

(33.3\%)

(59\%)

18

(30\%)

13

$(33.3 \%)$

0.17

$7(11 \%)$

27 (69\%)

$11(28 \%)$

50

(82\%)

$1(3 \%)$

0.027

$11 \quad 18(67 \%)$

(18\%)

$0(0 \%)$ 
*IQR - Interquartile range

*LTFU - Lost to follow-up

\section{Partner Support}

Among the 41 treated participants who had a partner, $78 \%(n=32)$ reported they personally asked their partner to be part of the treatment process. Among the 9 women who did not ask their partner to be part of the treatment process, $56 \%(n=5)$ reported their partner would have helped them get treatment had they asked (Table 4).

During the IDIs, women's comments on whether partners were supportive in encouraging and/or enabling women to seek treatment were conflicting, with some women reporting receiving support and others reporting a lack of support. When women talked about partners being supportive, it was usually in the context of providing money for transportation to the treatment facility. Some treated women said their husbands either encouraged them to seek treatment or accompanied them to the treatment facility:

"P: When I received the SMS that I tested positive, I shared that with my husband, and I could observe fear in him. I was brave to tell him that it's a normal disease like the others and it can be treated. So that compelled me to seek treatment as fast as possible.

l: What else encouraged you to seek treatment?

P: I shared with my husband my treatment appointment date, and he was very happy about that. The only challenge we had was lack of money to get me to Migori, [treatment hospital] so I explained to him the challenge of missing treatment appointment, and he immediately promised to borrow that money from a friend. That was so gracious of him."

Most women felt that they must rely on their husbands for transportation funds because women did not earn enough income to support that. Some women felt that if they told their husbands ahead of time, he would be able to help with household duties and childcare. One LTFU woman explained the conflicting support dynamics saying, "It depends with an individual, some will only provide that money 
when you are almost dying. They get to their senses when their friends challenge them to be responsible. Others take it as their own responsibility, and my husband I'm sure will support me."

When women discussed lack of support from their partners, they usually attributed it to men being uninformed or misinformed about HPV. Due to their lack of knowledge of HPV, women felt that men were less inclined to encourage their partners to seek treatment and were less inclined to provide funds for transport to the treatment facility. Additionally, several women mentioned that if men knew they had to wait for sex for a while after treatment, they might not let their partner go to the treatment facility. A treated woman said, "You may share with him your test results, and because they are not that well informed about it, he will discourage you not to go for treatment."

Additionally, women cited polygamy as a reason for lack of partner support. A LTFU woman related her experience relying on her partner to provide transport costs saying, "You can't do that with a polygamous man, you have to have your own sources. He doesn't care or he's not aware of money issues affecting us. We are five of us; can you depend on a man like that? No. You have to have your own sources of income to support your family." When women talked about decision-making in the household, most treated women said their husbands are the primary decision maker, while more LTFU women reported being the primary decision maker.

Table 4: Partner support among women who sought treatment 
Has partner asked how he can support in terms of seeking treatment

Yes

$31(51 \%)$

No

$10(16 \%)$

No Partner

$20(33 \%)$

Participant asked for partner to be part of treatment process $(\mathrm{N}=41)$

$32(78 \%)$

Partner would have helped get treatment if participant had asked $(\mathrm{N}=9) \quad 5$ (56\%)

Where did participant get transportation fare from

Partner

Self

Other close relative

Other

Friend

Children

\section{Stigma}

Stigma within the community often came up overtly when participants brought up concerns that men and women in the community would mistake them as a prostitute for seeking treatment. A treated woman said, "We are facing a lot of challenges in our families because at time our men think we are going out to prostitute, so some of us even if they test positive will not even think of going for treatment because of such." One participant explained that HPV is so new that nobody is talking about it. Reports of negative influence or shame were limited among treated women, although a couple of participants mentioned that other women shared negative sentiments about treatment, such as "People can also discourage and stigmatize you not to seek treatment."

\section{Knowledge and fear}

Lack of information or misinformation about HPV and cervical cancer came up frequently in the IDIs. Women generally knew the risk involved with HPV, but some women struggled to understand the 
difference between having HPV and having cervical cancer. Women knew that cancer could not be cured and that if HPV is diagnosed early enough it can be treated, but they typically did not have knowledge of HPV beyond this. Women in both groups openly stated they did not have enough information on HPV and would like additional information and counseling. Many women reported that even though they had some counseling on HPV during screening, when they got their positive HPV test result, they immediately assumed they had cancer.

"P: [I was scared when] I was diagnosed with HPV.

I: Why were you scared?

P: By having HPV made me think that I had cervical cancer."

Fear emerged often as a barrier to treatment in both groups of women; in most cases, it was regarding as fear of dying after receiving their positive HPV diagnosis. Some women reported they feared the treatment process, specifically concerned it would be painful or lead to complications later.

\section{Proposed treatment facilitators}

During the IDIs treated and LTFU women provided personal reasons for seeking treatment, or reasons for wanting to seek treatment. Most treated women cited the influence of others as a reason for seeking treatment. This consisted of encouragement from peers, partners, neighbors, children, health professionals, and co-wives in polygamous households. One treated woman said, "What encouraged me were what my friend shared and the possibility that if the disease is detected early enough it can be treated, so I had to try my luck." On the other hand, very few LTFU women were encouraged by friends or relatives to seek treatment and instead reported wanting to be healthy as their reason for wanting to seek treatment. This was also mentioned among treated women:

"Engaging them in a group is more effective than dealing with individuals. I'm comfortable being with the others when I'm being engaged in this kind of issue. Being on your own is stressing a lot ... because we are always on our own after receiving that result, no nurse or doctor to talk to." 
All women recommended improving partner support for treatment: involving men in counseling with their wives and including men in conversations about cervical cancer were overwhelmingly encouraged. A LTFU woman said, "We should love each other; that will enable us to bear each other's burden, and if we can engage men, they'll understand that this disease does not come because of prostitution. So we should counsel couples together." Many women felt that if health professionals talked to men themselves about the risks of cervical cancer, men would be more receptive to the information than if it came only from their wives.

In addressing stigma, treated women encouraged healthcare providers to protect patients' privacy, to make sure treatment is uniform across all patients, and to support and counsel women to access treatment. LTFU women more often discussed addressing stigma and recommended bringing facilities and services closer to them to keep their status private. Additionally, LTFU women felt that continued sharing of their treatment experiences would help eliminate stigma related to HPV in the community.

Providing money for transportation, providing a vehicle to shuttle women to the hospital in groups, and bringing the health services closer to them were overwhelmingly the most highly recommended treatment facilitators, although these did not differ across treated and LTFU women. Additionally, women recommended delivering test results and treatment instructions through a door-to-door format, rather than over SMS or phone call, to ensure the message makes it to everyone.

\section{Discussion}

This study contributes to cervical cancer prevention efforts by exploring the myriad reasons women who screen positive for HPV do or do not seek treatment. Findings highlight the need to address the substantial logistical barriers women face when seeking follow-up medical visits for cervical cancer treatment and prevention; however, these logistical barriers were faced by both women who did and did not seek treatment. We found that between two groups of women with very similar sociodemographic characteristics, social support, willingness to disclose HPV results and partner involvement seemed to play a pivotal role in their ability to seek treatment.

The barriers of cost of transportation and distance to the treatment facility were seen among treated and LTFU women, which was consistent with several other studies in similar settings. [5, 11-13] Reflecting this finding, the most commonly proposed facilitators to treatment seeking involved bringing health services closer to the community or providing transportation. Other similarities in observed barriers between treated and LTFU women included lack of partner support, stigma within the community, and feelings of stress and fear; similar findings have been reported previously in other studies.[12, 13, 14, 17]. These may 
be addressed by what women suggested as a potential facilitator: increased counseling on both the disease and treatment process. Women further added that counseling that included men would likely increase male involvement. Counseling and other strategies to reduce stigma could potentially increase partner support and decrease feelings of stress and fear related to their diagnosis.

Social support appears to be a dominant difference between the two groups of women and therefore may be a key factor to consider in encouraging follow-up. We found that treated women were significantly more likely to have peer support, which we saw when woman reported sharing their test results with other women. This finding was similar to that in other studies, which found that lack of social support was a potential barrier to follow-up care.[15, 16] Lack of social support was a clear barrier among LTFU women and a clear proposed facilitator to treatment among all women. Using social support as a facilitator for treatment seeking has been proposed in other studies in the past, supporting this finding. $[5,13]$ Overall, partner support dynamics were mostly similar between the two groups, although women collectively agreed that including partners in educational sessions about HPV would help garner support for treatment seeking, and eliminate misconceptions partners have about the disease. This finding was similar to other studies that showed women generally need approval or support from their partners to access health services. $[6,15]$ Similar to above, outreach and counseling interventions that incorporate stigma reduction could have a positive impact on partner support.

\section{Strengths and limitations}

A strength of this study is the use of qualitative data from rural women in East Africa, giving voice to their emotions and experiences in a way that allows them to shape healthcare delivery models. Another strength is the use of mixed methods, including the use of the survey questionnaire to inform selection of women for in-depth interviews based on their survey responses. Our study did have a few limitations, including the relatively small sample size for our survey data, which may have prevented the determination of statistically significant differences. Additionally, we only included women who were located in Migori County; therefore, generalization of the results is limited. Our results were similar to findings in other locations, so it is possible this information is generalizable to other low-resource settings.

\section{Conclusion}

Identification of the multiple barriers and facilitators to treatment seeking among HPV-positive women has the potential to reduce the substantial loss to follow-up for treatment. The results from this study suggest that there are complex barriers to treatment seeking among women in rural western Kenya that extend beyond the logistical challenges. Treated women expressed their relief in being able to successfully access treatment, and LTFU women expressed eagerness to get to the treatment facility. There is a need to develop new interventions that are able to overcome the substantial barriers women in rural communities face when accessing treatment after a positive HPV screen. Future interventions should aim to address logistical concerns, increase educational awareness among all community 
members, use social support as a means to address women in groups, and facilitate peer encouragement as a driver of health-seeking behavior. Little research has been conducted on perceived personal and social barriers and facilitators to follow-up treatment following a positive HPV screen, as this is a relatively new screening strategy in LMICs. Further research is needed to determine the relative contribution of the numerous factors examined in this study.

\section{Abbreviations}

HPV

Human-papillomavirus

LTFU

Lost to follow-up

KEMRI

Kenya Medical Research Institute

TAB

Treatment acquisition behavior

IDI

In-depth interview

LMIC

Low and middle-income countries

WHO

World Health Organization

\section{Declarations}

\section{Ethics approval and consent to participate}

The study received ethical approval from the Kenya Medical Research Institute (KEMRI) and Duke University. All women provided written or oral informed consent for participation in the survey and indepth interviews.

\section{Consent for publication}

Not applicable

\section{Availability of data and material}

The datasets used and/or analyzed during the current study are available from the corresponding author on request. 


\section{Competing interests}

The authors declare that they have no competing interests.

\section{Funding}

The research project on which these results are based received funding from $\mathrm{NIH}$-funded (R01 CA188248). BNK received support from the National Institute of Mental Health of the National Institutes of Health under award F32MH113288.

\section{Authors' contributions}

$\mathrm{MH}, \mathrm{SY}, \mathrm{SI}$ and BM participated in the design of the research and field data collection. CN participated in the design of the research, field data collection, analyzed the data, and drafted the manuscript. BK and LP advised data analysis, qualitative and quantitative, respectively. All authors contributed to editing the manuscript.

\section{Acknowledgements}

The Duke Master of Science in Global Health thesis committee for their guidance and support, as well as all Kenyan field research assistants for their hard work and perseverance, and foremost the study participants who dedicated their time to share their stories with us.

\section{References}

1. Bray, F., Ferlay, J., Soerjomataram, I., Siegel, R.L., Torre, L.A. and Jemal, A. (2018), Global cancer statistics 2018: GLOBOCAN estimates of incidence and mortality worldwide for 36 cancers in 185 countries. CA: A Cancer Journal for Clinicians, 68: 394-424. doi:10.3322/caac.21492

2. L B, L B-R, G A, B S, M M, D Gm, et al. ICO Information Centre on HPV and Cancer (HPV Information Centre). Human Papillomavirus and Related Diseases in Kenya. Summary Report 27 July 2017. 2017. Available from: http://www.hpvcentre.net/statistics/reports/KEN_FS.pdf.

3. Jemal A, Bray F, Center MM, Ferlay J, Ward E, Forman D. Global cancer statistics. CA Cancer J Clin. 2011;61(2):69-90. Epub 2011/02/08. doi: 10.3322/caac.20107. PubMed PMID: 21296855.

4. Finocchario-Kessler S, Wexler C, Maloba M, Mabachi N, Ndikum-Moffor F, Bukusi E. Cervical cancer prevention and treatment research in Africa: a systematic review from a public health perspective. 
BMC Womens Health. 2016;16:29. Epub 2016/06/05. doi: 10.1186/s12905-016-0306-6. PubMed PMID: 27259656; PubMed Central PMCID: PMCPMC4893293.

5. Paul P, Winkler JL, Bartolini RM, Penny ME, Huong TT, Nga le T, et al. Screen-and-treat approach to cervical cancer prevention using visual inspection with acetic acid and cryotherapy: experiences, perceptions, and beliefs from demonstration projects in Peru, Uganda, and Vietnam. Oncologist. 2013;18 Suppl:6-12. Epub 2013/12/18. doi: 10.1634/theoncologist.18-S2-6. PubMed PMID: 24334477.

6. Organization WH. Cancer Country Profiles, 2014. Available from: http://www.who.int/cancer/countryprofiles/ken_en.pdf?ua=1.

7. Organization WH. Comprehensive Cervical Cancer Control: A guide to essential practice; second edition. 2014. Available from: http://apps.who.int/iris/bitstream/10665/144785/1/9789241548953_eng.pdf.

8. Kuhn L, Denny L. The time is now to implement HPV testing for primary screening in low resource settings. Prev Med. 2017;98:42-4. Epub 2017/03/11. doi: 10.1016/j.ypmed.2016.12.030. PubMed PMID: 28279263; PubMed Central PMCID: PMCPMC5578476.

9. Ogilvie G, Nakisige C, Huh WK, Mehrotra R, Franco EL, Jeronimo J. Optimizing secondary prevention of cervical cancer: Recent advances and future challenges. Int J Gynaecol Obstet. 2017;138 Suppl 1:15-9. Epub 2017/07/12. doi: 10.1002/ijgo.12187. PubMed PMID: 28691338.

10. Huchko MJ, Ibrahim S, Blat C, Cohen CR, Smith JS, Hiatt RA, et al. A Cluster-Randomized Trial of HPV-based Cervical Cancer Screening in Community Health Campaigns Versus Health Facilities in Rural Western Kenya.

11. Percac-Lima S, Aldrich LS, Gamba GB, Bearse AM, Atlas SJ. Barriers to follow-up of an abnormal Pap smear in Latina women referred for colposcopy. J Gen Intern Med. 2010;25(11):1198-204. Epub 2010/07/24. doi: 10.1007/s11606-010-1450-6. PubMed PMID: 20652647; PubMed Central PMCID: PMCPMC2947627.

12. Vastbinder MB, Castillo C, Bekkers RL. Barriers to follow-up of abnormal Papanicolaou smears among women in Leon, Nicaragua. Trop Doct. 2010;40(1):22-6. Epub 2009/12/17. doi: 10.1258/td.2009.090076. PubMed PMID: 20008060.

13. Bingham A, Bishop A, Coffey P, Winkler J, Bradley J, Dzuba I, et al. Factors affecting utilization of cervical cancer prevention services in low-resource settings. Salud Publica Mex. 2003;45 Suppl 3:S408-16. Epub 2004/01/30. PubMed PMID: 14746034.

14. Aharon D, Calderon M, Solari V, Alarcon P, Zunt J. Barriers to Follow-Up for Abnormal Papanicolaou Smears among Female Sex Workers in Lima, Peru. PLoS One. 2017;12(1):e0169327. Epub 2017/01/07. doi: 10.1371/journal.pone.0169327. PubMed PMID: 28060937; PubMed Central PMCID: PMCPMC5217960.

15. Garces-Palacio IC, Ramos-Jaraba SM, Rubio-Leon DC. Health Beliefs Associated with the Follow-Up of Pap Smear Abnormalities Among Low-Income Women in Medellin, Colombia. J Cancer Educ. 2017. Epub 2017/01/26. doi: 10.1007/s13187-017-1172-0. PubMed PMID: 28120138. 
16. Abercrombie PD. Improving adherence to abnormal Pap smear follow-up. J Obstet Gynecol Neonatal Nurs. 2001;30(1):80-8. Epub 2001/03/30. PubMed PMID: 11277165.

17. Binka C, Nyarko SH, Awusabo-Asare K, Doku DT. Barriers to the Uptake of Cervical Cancer Screening and Treatment among Rural Women in Ghana. Biomed Res Int. 2019;2019:6320938. Published 2019 Nov 3. doi:10.1155/2019/6320938 